\title{
Central Airway Obstruction Due to Tracheal Glomus Tumor
}

\author{
Greg J. Haro, $\mathrm{MD}^{1} \quad$ Eric J. Seeley, $\mathrm{MD}^{2} \quad$ David M. Jablons, $\mathrm{MD}^{1} \quad$ Johannes R. Kratz, MD ${ }^{1}$ \\ 1 Department of Surgery, Division of Cardiothoracic Surgery, University of \\ California, San Francisco; San Francisco, California, United States \\ 2 Department of Medicine, Division of Pulmonary \& Critical Care, \\ University of California, San Francisco; San Francisco, California, \\ United States

\begin{abstract}
Address for correspondence Johannes R. Kratz, MD, Department of Cardiothoracic Surgery, University of California, San Francisco, 500 Parnassus Avenue Suite MUW-405, San Francisco, CA 94143-0118,
\end{abstract} \\ United States (e-mail: johannes.kratz@ucsf.edu).
}

Thorac Cardiovasc Surg Rep 2018;7:e43-e45.

\author{
Abstract \\ Keywords \\ - glomus tumor \\ - tracheal glomus \\ tumor \\ - central airway \\ obstruction
}

Background Tracheal glomus tumors are rare mesenchymal neoplasms that have the potential to cause malignant, central airway obstruction. They require a thoughtful approach to safely secure the airway and definitively resect the tumor.

Case Description We report the clinical course of a 25-year-old man in severe respiratory distress secondary to tracheal glomus tumor and the subsequent surgical management. Conclusion Due to their hypervascular nature, greater familiarity with tracheal glomus tumors is needed to ensure appropriate preoperative planning and intervention.

\section{Introduction}

Glomus tumors are rare neoplasms that arise from the glomus apparatus, which is a specialized form of arteriovenous shunt involved in thermoregulation. ${ }^{1}$ They most commonly occur in the subungual space and dermis; however, they can occur throughout the body including bone, stomach, lung, mediastinum, and trachea. ${ }^{1,2}$ From these possible sites, tracheal glomus tumors are the most clinically significant as they can cause malignant, central airway obstruction. We report the presentation of a patient with hemoptysis requiring urgent intervention to secure the airway and the subsequent surgical management.

\section{Case Description}

A 25-year-old man with extreme obesity reported a 1-year history of cough and was treated for asthma without relief of his symptoms. He subsequently developed hemoptysis prompting computed tomography that revealed a $3.6 \times 3.9 \times 4.9 \mathrm{~cm}$ mass within the wall of the trachea that caused critical narrowing of the trachea ( - Fig. 1 ).

The patient was found to be in near extremis with inspiratory stridor and was taken to the operating room urgently to secure the airway with bronchoscopic debridement. Awake anesthesia was performed, and a flexible bronchoscope was used to pass a 5-0 endotracheal tube distal to the tumor. The right internal jugular vein was then percutaneously cannulated with a 31-Fr Avalon Elite catheter (Maquet, Wayne, New Jersey, United States) under fluoroscopic and echocardiographic guidance and veno-venous extracorporeal membrane oxygenation (ECMO) was then initiated. The tumor was biopsied via rigid bronchoscopy and thereafter bled extensively. Despite application of neosynephrine and argon coagulation, bleeding only ceased following passage of an 8-0 endotracheal tube distal to the tumor.

Further attempt at bronchoscopic debridement was aborted, and the patient was decannulated and returned to the intensive care unit intubated. On review of the pathology (-Fig. 2), the tumor was composed of nests of monotonous round cells and thin-walled vessels containing focally infiltrating smooth muscle. Immunohistochemistry stains were positive for smooth muscle actin and negative for chromogranin. While rare, these findings suggested tracheal glomus tumor.

The patient was then brought back to the operating room for definitive resection. Repeat flexible bronchoscopy revealed that the tracheal tumor was located approximately $4 \mathrm{~cm}$ distal to the larynx and extended to $4 \mathrm{~cm}$ proximal to the carina. A collar incision with an upper partial median sternotomy was performed to expose the tumor fully. The received

July 7, 2018

accepted after revision

September 24, 2018
DOI https://doi.org/

10.1055/s-0038-1675411. ISSN 2194-7635. (c) 2018 Georg Thieme Verlag KG
Stuttgart · New York

License terms

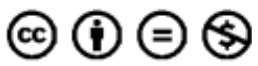




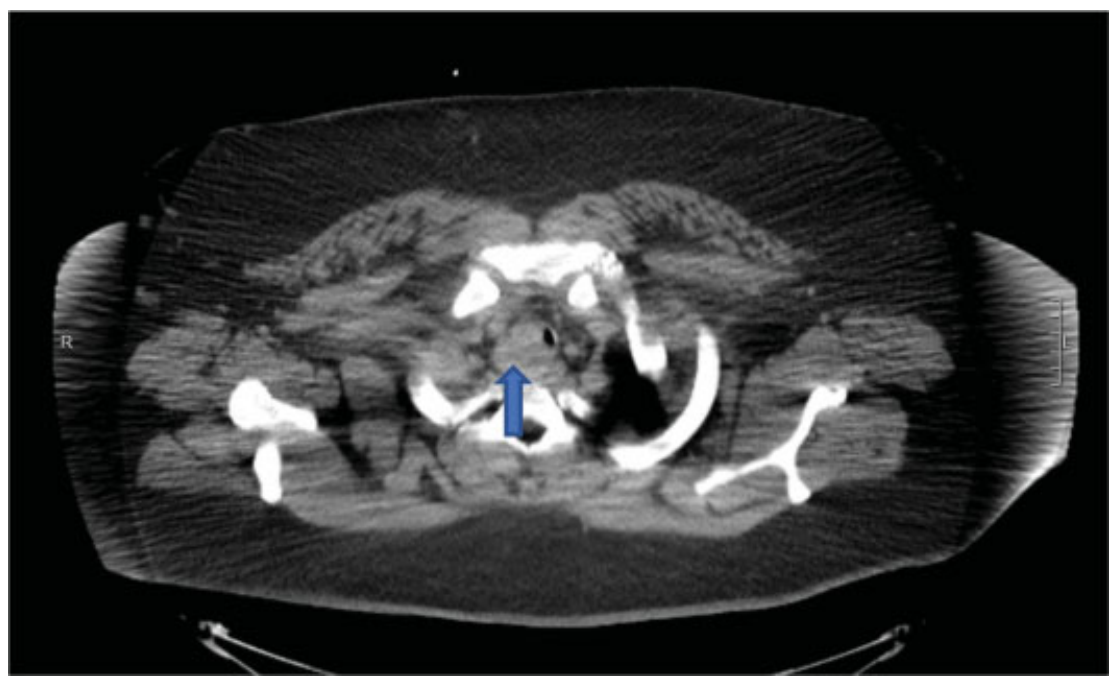

Fig. 1 Preoperative computed tomography of the neck/chest. A $3.6 \times 3.9 \times 4.9 \mathrm{~cm}$ mass within the wall of the trachea at the cervicothoracic junction.

tumor was found to involve the right tracheal wall with extraluminal extension, but without invasion of the surrounding structures. The trachea was circumferentially dissected, and the tumor was excised ( - Fig. 3 ) with the use of cross-table ventilation and an armored endotracheal tube. The trachea was reconstructed end-to-end without the need for releasing maneuvers or endoscopic stenting. The left sternohyoid muscle was mobilized and placed between the tracheal anastomosis and the innominate artery. The patient was then extubated and returned to the intensive care unit.

Postoperatively, the patient developed pneumonia that required bronchoscopy, and the hospital course was otherwise unremarkable. In review at our multidisciplinary tumor board, the final diagnosis was glomus tumor of uncertain malignant potential given the deep location and size greater than $2 \mathrm{~cm}$. The surgical margins were negative. It was decided to pursue surveillance with computed tomography at 3,6, and 12 months and bronchoscopy at 12 months.

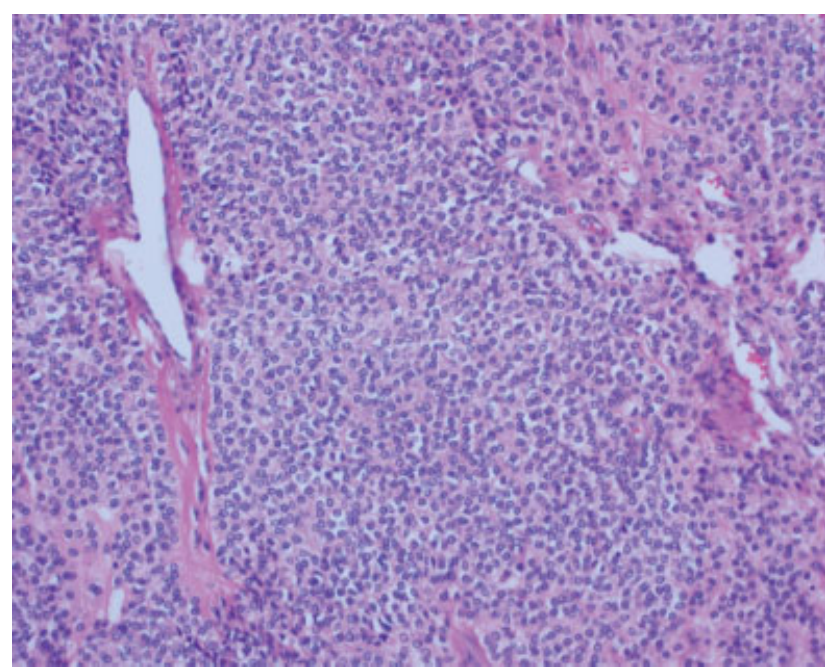

Fig. 2 Pathology. Nests of monotonous round cells with pale eosinophilic cytoplasm and thin-walled vessels containing focally infiltrating smooth muscle (hematoxylin/eosin, $20 \times$ ).

\section{Discussion}

Tracheal glomus tumors are hypervascular tumors that grow concentrically and can cause central airway obstruction. There have been approximately 70 case reports to date, and interestingly half of these reports have occurred in the past decade. ${ }^{2}$ It is unclear if there has been an increase in incidence or if there has been improved pathologic identification. They occur most commonly in the 4th and 5th decades of life with 2:1 male predominance, ${ }^{2}$ and they have the potential to be malignant. ${ }^{3}$ While almost half are located in the distal trachea, they can be found throughout the trachea. Up to $10 \%$ of these tumors are found incidentally, but patients usually are symptomatic with dyspnea, cough, and/or hemoptysis. ${ }^{4}$

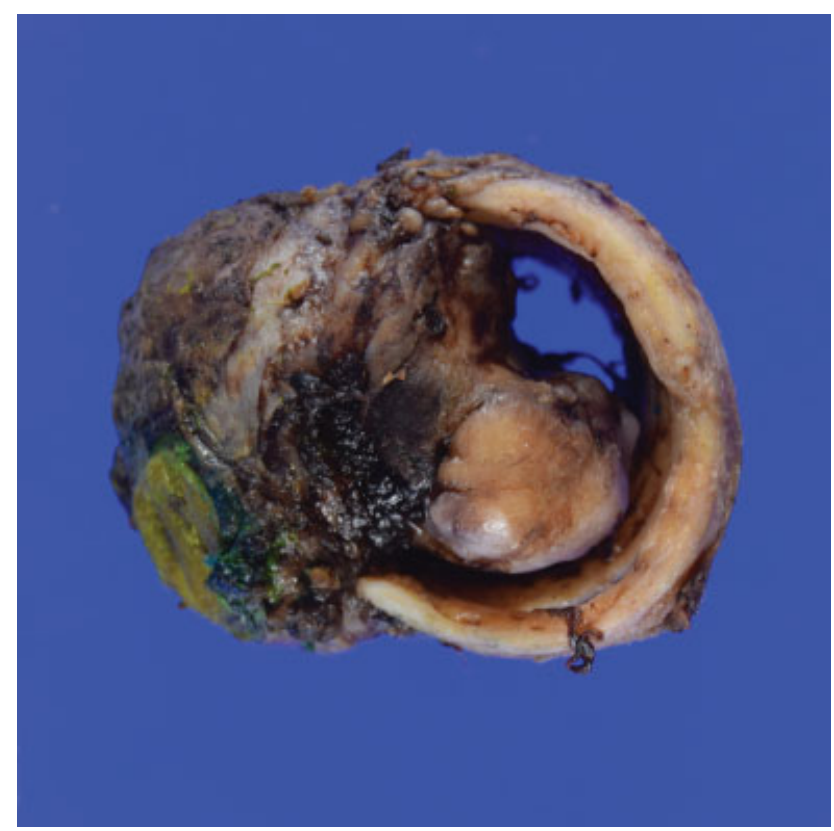

Fig. 3 Tumor specimen. Concentric growth of tumor causing central airway obstruction. 
In patients with central airway obstruction, careful preparation and use of a diverse repertoire of techniques to ensure a successful procedure is essential. Our approach is to first assess the presumptive diagnosis, the severity and location of obstruction, the overall clinical state of the patient, and any patient factors that would prevent a successful procedure. From this assessment, we then determine if standard techniques will be sufficient, such as rigid bronchoscopy with jet insufflation, or if more advanced support will be required, such as ECMO. In regard to our patient, our differential diagnosis was broad and included both benign and malignant etiologies. The tumor was near occlusive at the mid-trachea, the patient had severe respiratory distress with hemoptysis, and there was heightened concern regarding the extreme obesity. Due to these factors, ECMO was utilized as an adjunctive safety measure.

The most salient lesion to be learned from this case is the hypervascular nature of glomus tumors. Although our initial goal was bronchoscopic biopsy and debridement, the tumor bled extensively following biopsy. Due to our preparedness, ECMO afforded us the time to stop the bleeding, while maintaining appropriate oxygenation. In review of the imaging, there were moderately sized vessels feeding the tumor and the patient may have benefited from preoperative embolization of the tumor. Although hypervascular tracheal tumors are rare, we have changed our practice in that we more closely consider preoperative embolization of large tracheal tumors causing central airway obstruction to reduce the risk of bleeding and increase the prospect of successful intervention.

In conclusion, tracheal glomus tumors are a rare cause of malignant central airway obstruction. Due to their hypervascular nature, greater familiarity with tracheal glomus tumors is needed to ensure appropriate preoperative planning and intervention.

\section{Funding}

None.

\section{Acknowledgment}

None.

\section{References}

1 McDermott EM, Weiss AP. Glomus tumors. J Hand Surg Am 2006; 31(08):1397-1400

2 Wang C, Ma Y, Zhao X, et al. Glomus tumors of the trachea: 2 case reports and a review of the literature. J Thorac Dis 2017;9(09): E815-E826

3 Huang C, Liu QF, Chen XM, et al. A malignant glomus tumor in the upper trachea. Ann Thorac Surg 2015;99(05):1812-1814

4 Gowan RT, Shamji FM, Perkins DG, Maziak DE. Glomus tumor of the trachea. Ann Thorac Surg 2001;72(02):598-600 